\title{
Clinical Evaluation of a Novel Blended Mode Diode Laser for Hair Removal
}

\author{
Michael H. Gold1, Judith Hellman'2, \\ Serge Dahan ${ }^{3}$, R. Stephen Mulholland4* \\ ${ }^{1}$ Tennessee Clinical Research Center, Nashville, USA \\ ${ }^{2}$ Mt. Sinai Hospital, New York, USA \\ ${ }^{3}$ Jean Languedoc Clinic, Toulouse, France \\ ${ }^{4}$ Private Aesthetic Plastic Surgery Practice, SpaMedica, Toronto, Canada \\ Email: *mulhollandmd@spamedica.com
}

How to cite this paper: Gold, M.H., Hellman, J., Dahan, S. and Mulholland, R.S. (2019) Clinical Evaluation of a Novel Blended Mode Diode Laser for Hair Removal. Journal of Cosmetics, Dermatological Sciences and Applications, 9, 19-29. https://doi.org/10.4236/jcdsa.2019.91002

Received: December 18, 2018

Accepted: January 20, 2019

Published: January 23, 2019

Copyright (C) 2019 by author(s) and Scientific Research Publishing Inc. This work is licensed under the Creative Commons Attribution International License (CC BY 4.0).

http://creativecommons.org/licenses/by/4.0/

\begin{abstract}
Introduction: Photoepilation by lasers is a popular procedure in aesthetic dermatology for removing unwanted body and facial hair. The use of the most appropriate laser wavelength is crucial as it affects treatment depth and melanin absorption. The three commonly used hair removal lasers are of specific wavelengths: $755 \mathrm{~nm}, 810 \mathrm{~nm}$, and $1064 \mathrm{~nm}$, each preferred certain types of skin and hair characteristics. The current evaluation reports the safety and efficacy of unique blended modes 755/810 nm and 810/1064 nm diode lasers for hair removal. Methods: Hair removal results from 50 patients treated with the 755/810 $\mathrm{nm}$ handpiece and 50 patients treated with the 810/1064 nm handpiece were gathered from a few clinics. 3 treatments on various body areas were conducted 6 weeks apart and patients were followed up with 6 months after the last treatment. Results were evaluated by baseline, follow-up photographs, and hair counts. Results: Treatment area photos demonstrated hair reduction in the treated body and facial areas. Average hair count reduction at 6 months follow-up was $84 \%$ for the $755 / 810 \mathrm{~nm}$ handpiece and $81 \%$ for the $810 / 1064 \mathrm{~nm}$ handpiece. No significant or unexpected adverse events were detected in any of the patients. Conclusions: The novel blended mode hair removal diode lasers are proven to be safe and effective for hair removal in facial and body areas for patients of various skin types and hair characteristics.
\end{abstract}

\section{Keywords}

Laser Hair Removal, 755 nm Diode Laser, 810 nm Diode Laser, $1064 \mathrm{~nm}$ Diode Laser 


\section{Introduction}

Laser hair removal treatment is based on the principle of selective photothermolysis. In this process, the absorbed optical energy is converted into heat, coagulating the hair follicle [1]. There are preferential wavelengths for melanin absorption. At three specific wavelengths $(755 \mathrm{~nm}, 810 \mathrm{~nm}$, and $1064 \mathrm{~nm})$, absorption by melanin is greater than competitive absorption by oxyhemoglobin and water. These wavelengths correspond to three popular hair removal lasers: 755 $\mathrm{nm}$ alexandrite, $810 \mathrm{~nm}$ diode, and $1064 \mathrm{~nm} \mathrm{Nd:YAG} \mathrm{lasers} \mathrm{[2].}$

Numerous published clinical studies have demonstrated the safety and efficacy of different lasers at various wavelengths for hair removal [1] [3].

Based on clinical evidence, hair removal diode lasers of $810 \mathrm{~nm}$ are considered as the universal hair removal for all skin types, and these lasers are commonly used in the market [1] [2] [3]. Light color or fine hair may be resistant to the treatment due to scarcity of pigmentation in the hair shaft, whereas treatment of dark skin type patients is difficult due to skin melanin absorption [1]. Light color and fine hair respond better to $755 \mathrm{~nm}$, while a wavelength of $1064 \mathrm{~nm}$ is more suitable for dark skin [2].

Recently new diode lasers of $755 \mathrm{~nm}$ and $1064 \mathrm{~nm}$ were developed to enable suitable diode laser treatments for a wide variety of skin and hair types [4] [5] [6].

This manuscript describes the evaluation of a newly developed device with two different handpieces generating optical energy from diode bars at 755 and $810 \mathrm{~nm}$ simultaneously and at 810 and $1064 \mathrm{~nm}$ simultaneously. The novel blended wavelength handpieces are applied to the skin for selective hair removal for a variety of hair and skin types.

This study presents results of a multicenter evaluation of the blended wavelength $755 / 810 \mathrm{~nm}$ and $810 / 1064 \mathrm{~nm}$ lasers for hair removal.

\section{Materials and Methods}

\section{The Device}

The InMode Triton/DiolazeXL device (InMode MD Ltd., Israel) is designed to deliver optical energy to the skin via a pre-cooled sapphire block. Good optical contact between the sapphire block and the skin is achieved by using water-based gel. The device provides individual adjustment of light fluence and pulse duration to achieve maximum efficiency and safety for each patient. The handpiece has integrated skin cooling to enhance safety and comfort of the treatment.

The device is shown in Figure 1.

The diode laser handpieces are available in 3 wavelength configurations:

- Mix of $755 \mathrm{~nm} / 810 \mathrm{~nm}$ and a mix of $810 \mathrm{~nm} / 1064 \mathrm{~nm} .810 \mathrm{~nm}$ alone is also available as the most commonly used wavelength, but it is not the subject of this study.

- Each of the handpieces are comprised of 20 diode laser bars stacked verti- 
cally. With the blended modes the corresponding diode bars are alternated and the two wavelengths of $755 / 810$ or $810 / 1064$ are emitted simultaneously through a chilled sapphire light guide.

A schematic representation of the alternating diode bars is illustrated in Figure 2.

The diode laser produces up to $3000 \mathrm{~W}$ peak optical power.

The sapphire light guide is located at the front of the handpiece and delivers the laser beam energy to the treated tissue, while cooling the skin. The sapphire block is surrounded by a chilled gold-plated metal frame. This structure provides increased cooling to a temperature of about $7^{\circ} \mathrm{C}$ on the treatment spot and a few millimeters around it. The handpiece cooling flow is demonstrated in Figure 3. The arrow indicates the tip progress direction on the skin. One part of the metal frame is responsible for pre-cooling, the sapphire light guide is responsible for the cooling stage and the other part of the metal frame is responsible for post-cooling during handpiece progression.

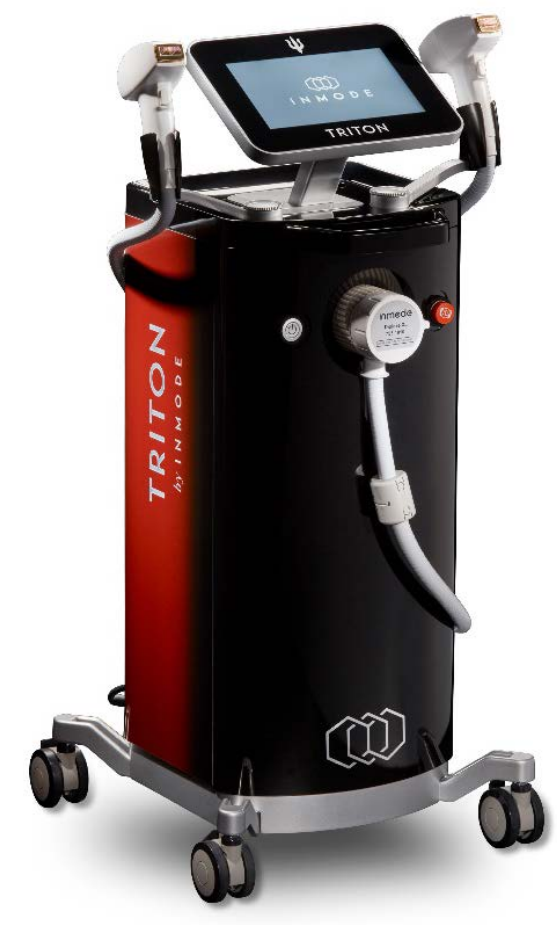

Figure 1. Triton/DiolazeXL Device.

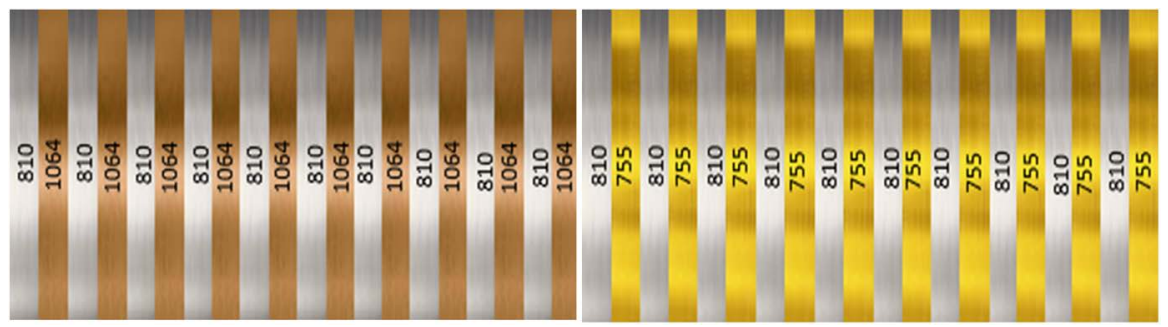

Figure 2. Illustration of the diode bars in the blended mode handpieces. 755/810 nm (left) and 810/1064 nm (right). 


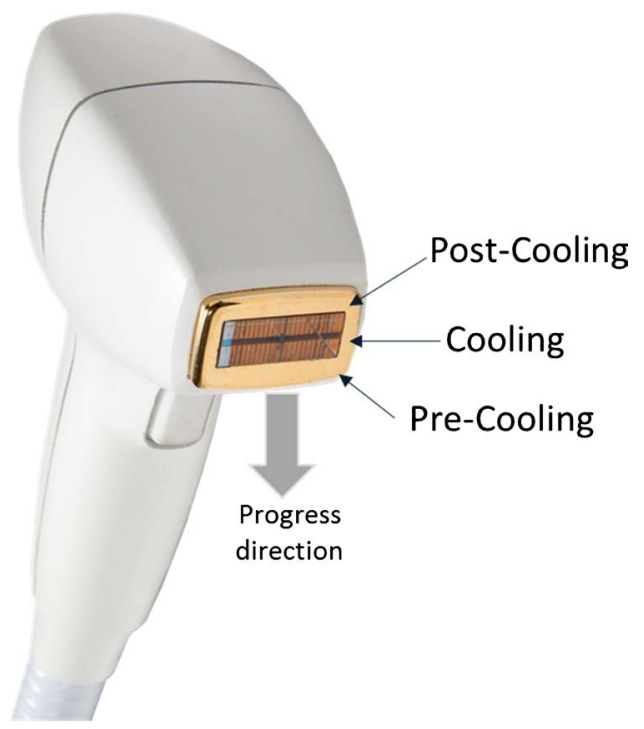

Figure 3. Handpiece tip cooling.

The dimensions of the sapphire light guide are $11 \mathrm{~mm} \times 27.5 \mathrm{~mm}$.

A temperature sensor is located on the enclosure of the sapphire cooler and measures the temperature of the tissue cooler to ensure stable sapphire temperature.

\section{Evaluation Design}

The objectives of this multicenter evaluation were to gather safety and efficacy hair removal data of the newly developed diode laser blended modes 755/810 nm and $810 / 1064 \mathrm{~nm}$ handpieces.

Results from 50 subjects treated with the $755 / 810 \mathrm{~nm}$ handpiece and 50 subjects who were treated with the $810 / 1064 \mathrm{~nm}$ handpiece were accumulated from a few clinics in the USA, Canada, France, and Israel during 2018.

Subjects underwent 3 treatment sessions 6 weeks apart and were followed up with 6 months post their last treatment.

Female and male subjects of Caucasian, Hispanic, Asian, and Afro-American backgrounds were treated on different areas such as the legs, underarms, bikini, chest, back and face.

The study efficacy was assessed by average hair reduction at the 6 month follow-up visit compared to the baseline count. Hair counts were performed on photographs taken from the subjects' treatment areas. Subjects consented to use of their photographs.

Safety of the device was evaluated by assessing the incidence, severity and persistence of adverse events, if any occurred during the study period.

\section{Photography}

Photos of the treatment areas were taken at baseline and at the 6 month follow-up visit in a reproducible manner. The hair was trimmed to $1-3 \mathrm{~mm}$ length and the area before trimming as well as the trimmed area was photographed.

Hair counts were performed by marking visible hairs on photographs of the subjects' treatment area. Figure 4 demonstrates counting examples. 

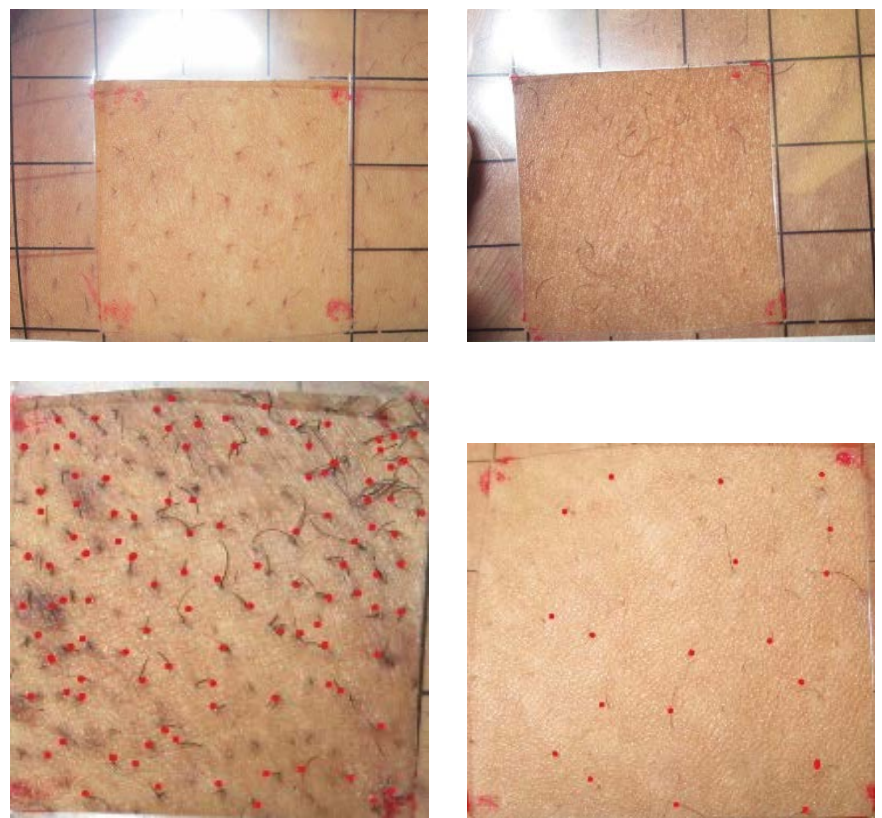

Figure 4. Examples of area marking in bikini (upper) and hair counts in underarm (lower) areas conducted in photos before (left) and at follow-up visit (right).

\section{Results}

Hair removal results from 50 subjects treated with the $755 / 810 \mathrm{~nm}$ laser and 50 subjects treated with the $810 / 1064 \mathrm{~nm}$ laser were included in the study. All subjects had completed all treatment and follow-up regimen.

\section{Results of Hair Removal Using the 755/810 nm Handpiece}

44 female subjects and 6 male subjects, who requested hair removal treatments, were treated with the $755 / 810 \mathrm{~nm}$ handpiece. The mean subject age was 25.4 with minimum and maximum age of 19 and 36, correspondingly. Subjects had the following Fitzpatrick skin types: type II (66\%) and type III (34\%).

Subjects were treated on various treatment areas for hair removal with the 755/810 $\mathrm{nm}$ blended mode hand piece including underarm, bikini, legs, face, men's back, and chest.

Analysis of hair count change from baseline to the 6 month follow-up (Table 1) resulted in a weighted average hair count reduction of $84 \%$ (range of $75 \%$ $88 \%)$.

Representative photos of various areas treated with the 755/810 nm diode laser handpiece taken at baseline and at 6 month follow-up time points are presented below in Figures 5-7.

\section{Results of Hair Removal Using the 810/1064 nm Handpiece}

50 female subjects, who were interested in hair removal treatments, were treated with the $810 / 1064 \mathrm{~nm}$ handpiece. The mean subject age was 24 with a range of 19 - 36. Subjects had the following Fitzpatrick skin types: type IV (37.5\%), type V (41.5\%) and type VI (21\%).

Subjects were treated on various treatment areas for hair removal with the 810/1064 nm blended mode handpiece, including underarm, bikini, legs, and face. 
Analysis of hair count change from baseline to the 6 month follow-up resulted in an average weighted hair count reduction of $81 \%$ (range of $70 \%-84 \%$ ). (Table 2).

Table 1. Weighted average hair count reduction according to different treatment areas.

\begin{tabular}{ccc}
\hline Treatment area & No. & Average \% reduction \\
\hline Underarm & 12 & 88 \\
Bikini & 13 & 86 \\
Leg & 13 & 83 \\
Face & 6 & 75 \\
Back & 3 & 78 \\
Chest & 3 & 77 \\
Total & 50 & 84
\end{tabular}

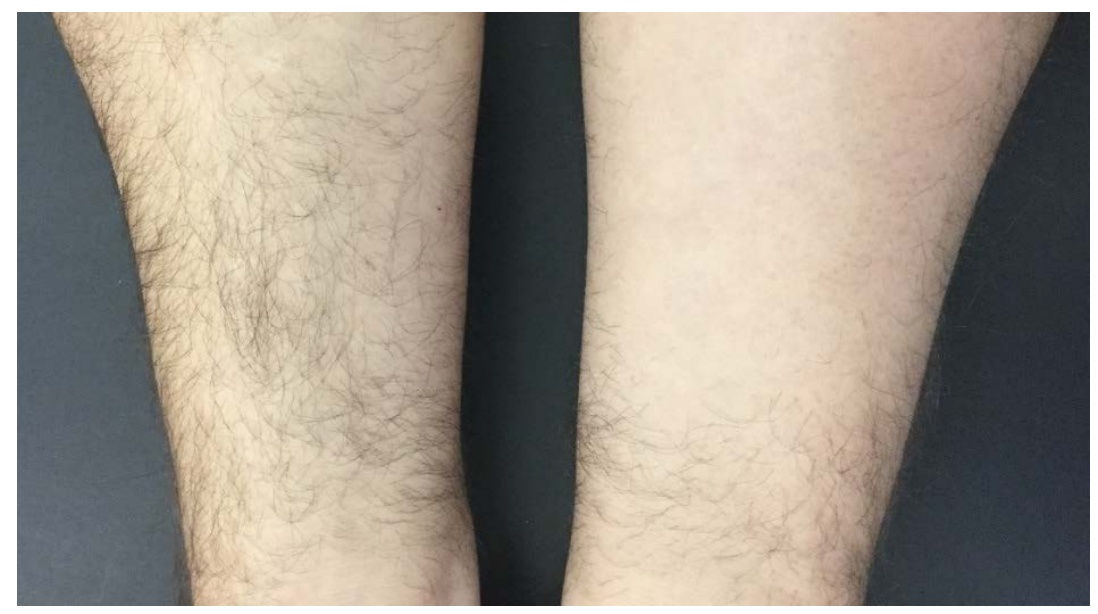

Figure 5. Arm treated with 755/810 handpiece at baseline (left) and at 6 months after last treatment (right).

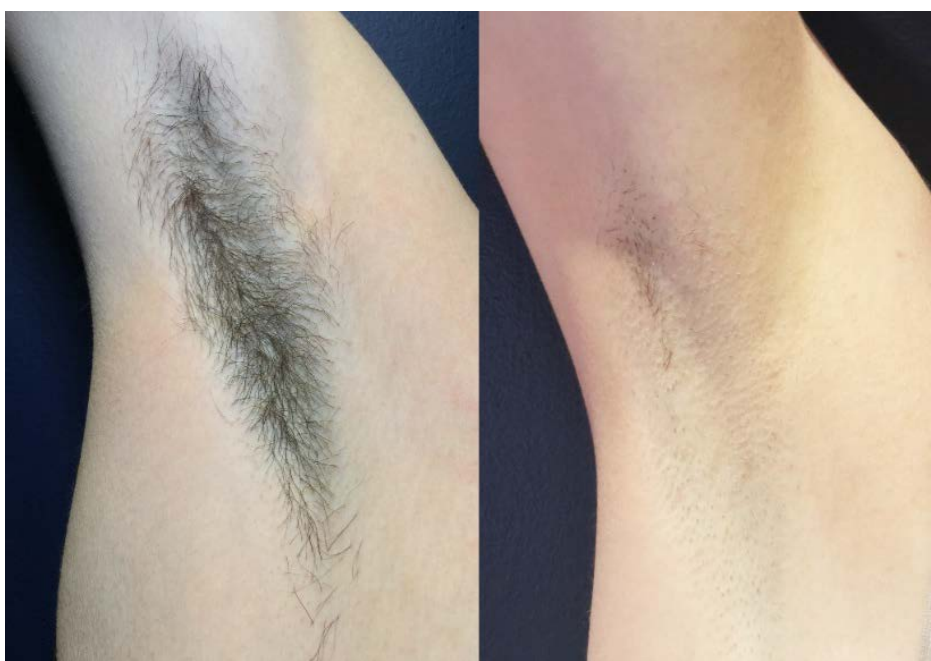

Figure 6. Underarm treated with $755 / 810$ hand piece at baseline (left) and at 6 months after last treatment (right). 
Representative photos of various areas treated with the 810/1064 $\mathrm{nm}$ diode laser handpiece taken at baseline and at 6 months follow-up time points are presented below in Figures 8-10.

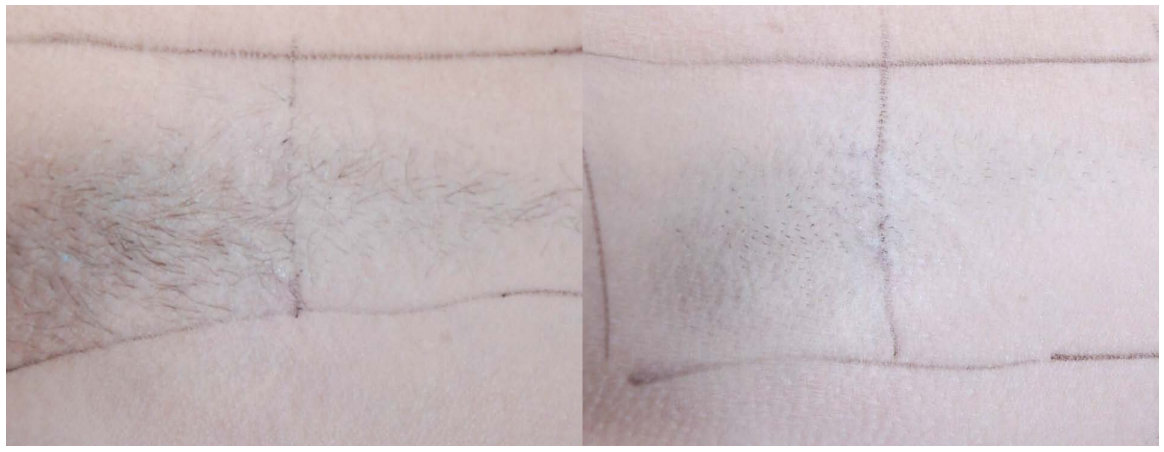

Figure 7. Underarm treated with $755 / 810$ hand piece at baseline (left) and at 6 months after last treatment (right).

Table 2. Weighted average hair count reduction according to different treatment areas.

\begin{tabular}{ccc}
\hline Treatment area & No. & Average \% reduction \\
\hline Underarm & 14 & 84 \\
Bikini & 15 & 82 \\
Leg & 15 & 81 \\
Face & 6 & 70 \\
Total & 50 & 81 \\
\hline
\end{tabular}

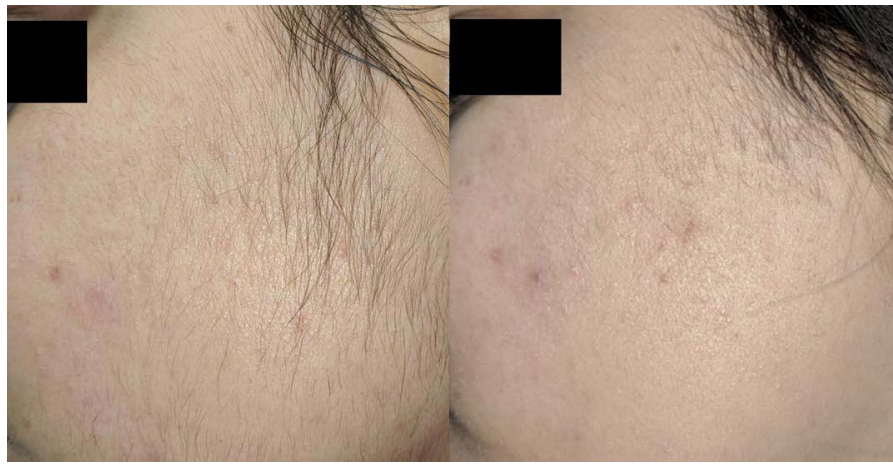

Figure 8. Face of a dark skin type subject treated with 810/1064 handpiece at baseline (left) and at 6 months after last treatment (right).
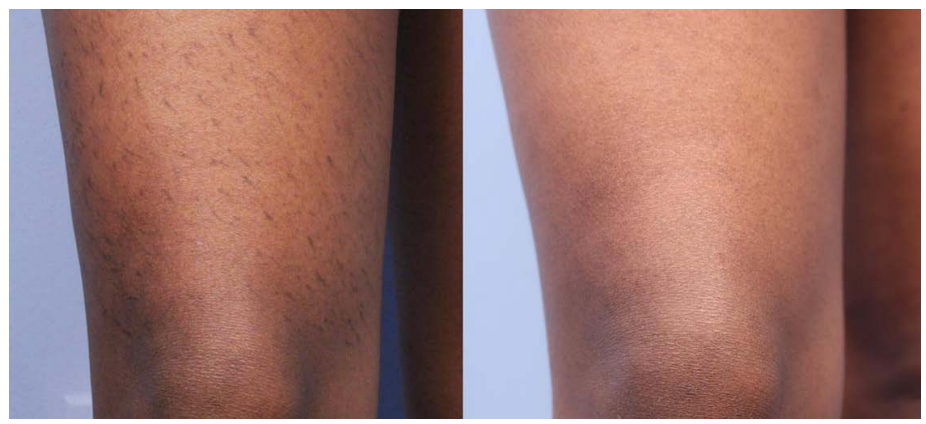

Figure 9. Knee of a dark skin type subject treated with 810/1064 handpiece at baseline (left) and at 6 months after last treatment (right). 


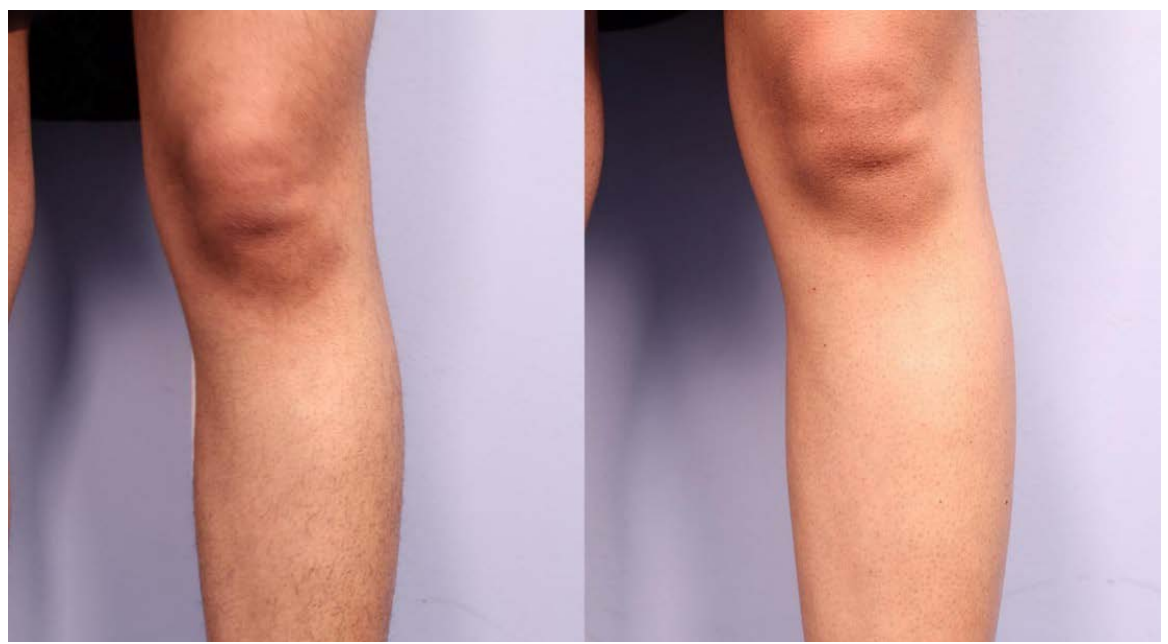

Figure 10. Leg of a dark skin type subject treated with 810/1064 hand piece at baseline (left) and at 6 months after last treatment (right).

\section{Safety Analysis}

No unexpected or severe adverse events were reported. Mild erythema and edema that appeared following the treatment subsided shortly.

\section{Discussion}

The choice of an optimal laser for the individual patient is critical for treatment success and safety. One should take into consideration the patient's skin and hair types to match laser wavelength that will reflect melanin absorption and penetration depth.

The wavelength range of 650 to $1300 \mathrm{~nm}$ is considered generally suitable for typical depth of hair from $1 \mathrm{~mm}$ to $3 \mathrm{~mm}$. The best penetration depth is around $1060 \mathrm{~nm}$ corresponding to the Nd:YAG laser, but this wavelength absorption in hair melanin is relatively low and efficacy of treatment is not very high. The maximal efficacy is around $650-700 \mathrm{~nm}$ but penetration depth is limited at this wavelength.

A wavelength of $755 \mathrm{~nm}$ is considered very effective for light skin treatment [4] while the $1064 \mathrm{~nm} \mathrm{Nd:YAG} \mathrm{laser} \mathrm{became} \mathrm{popular} \mathrm{for} \mathrm{hair} \mathrm{removal} \mathrm{on} \mathrm{dark}$ skin patients [5] [6].

One of the compromises between these two ranges is the use of an $810 \mathrm{~nm}$ diode [7] [8] [9] [10] [11] which has better penetration depth than $755 \mathrm{~nm}$ and much higher absorption coefficient of melanin than the Nd:YAG laser. Sometimes better tuning of the wavelength is optimal, especially for darker skin and non-black hairs.

For dark skin it would be a logical step to use a wavelength between $810 \mathrm{~nm}$ and $1064 \mathrm{~nm}$, but the peak of light absorption by water in this range makes these wavelengths less effective for treatment. In addition, not all wavelengths are available in the market because of technological limitations.

One of the solutions is to use a combination of two wavelengths, which are al- 
ready well-known and clinically proven for laser hair removal treatment.

The use of combination $810 \mathrm{~nm}$ and $1064 \mathrm{~nm}$ would improve penetration depth and safety for darker skin while maintaining relatively high efficacy of treatment.

Combination of $755 \mathrm{~nm}$ and $810 \mathrm{~nm}$ would allow optimization of treatment for different hair colors.

Because all these wavelengths are recognized as safe and effective for hair removal the combination of the wavelengths does not seem to raise any safety concerns and enables efficacy optimization.

The novel diode laser Triton/DiolazeXL with the blended mode of wavelengths, $755 / 810 \mathrm{~nm}$ and $810 / 1064 \mathrm{~nm}$, enables a wide range of penetration depths and hair melanin absorption. Figure 11 illustrates the unique penetration of the two blended mode lasers.

Clinical experience supports the safety and efficacy of both handpieces for hair removal on light and dark skin in various body and facial areas with a range of hair types.

The current evaluation demonstrated treatment results from female and male subjects who underwent 3 treatment sessions 6 weeks apart and were followed up with 6 months following their last treatment. As expected, due to better absorption in melanin of the lower wavelength, subjects treated with 755/810 nm hand piece displayed slightly better hair reduction results (average reduction of $84 \%$ ) than those treated with $810 / 1064 \mathrm{~nm}$ handpiece (average reduction of $81 \%)$. When comparing different treatment areas, underarms presented the best hair reduction, as expected from relatively lighter skin and coarser, darker hair in these areas. The same applies to bikini areas, but to a slightly lesser degree. The legs usually have finer hair than underarm and bikini, and exhibited less hair reduction, as well as deeper hair on men's back and chest. The lowest percent of hair reduction was expressed on the face due to its very fine hair with a

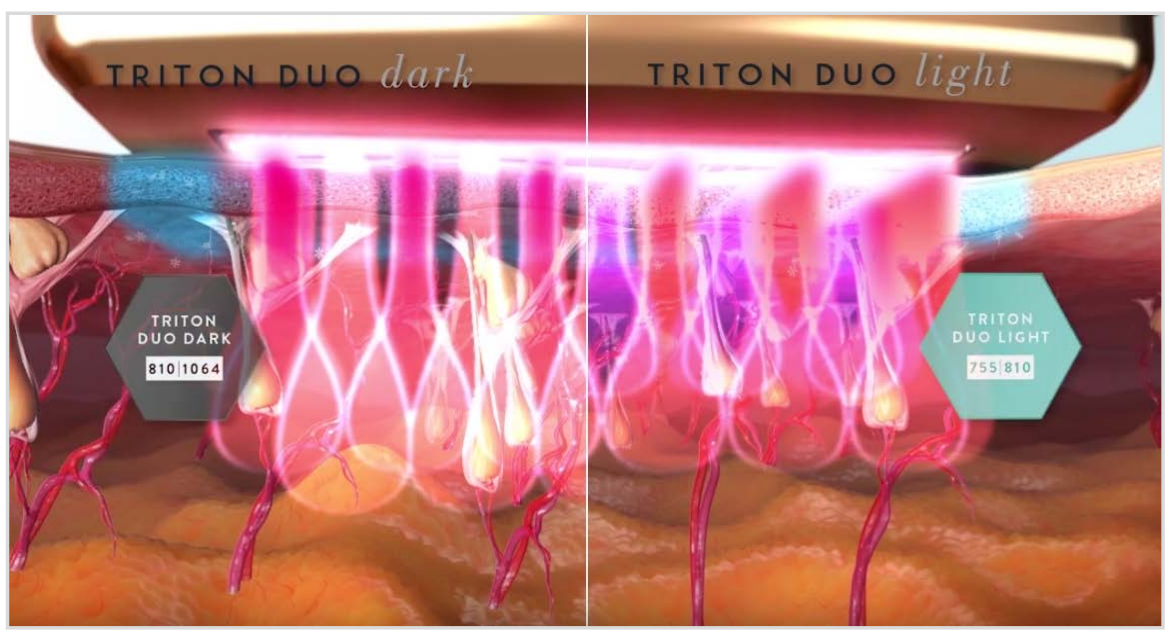

Figure 11. An illustration of the interaction between the blended mode lasers and the hair structure. 810/1064 (left) and 755/810 (right) at various depths (InMode proprietary material). 
limited amount of melanin. All the above is applicable to both handpieces, $755 / 810 \mathrm{~nm}$ and $810 / 1064 \mathrm{~nm}$.

No unexpected adverse events were reported in this study.

The clinical data presented here demonstrate that the unique blended mode of wavelengths, $755 / 810 \mathrm{~nm}$ and $810 / 1064 \mathrm{~nm}$, has proven to be safe and effective for hair removal on light and dark skin.

The novel combination approach enables more accurate and personalized treatment, thus maximizing hair removal results while minimizing treatment risks. More studies are planned to further substantiate the potential clinical contribution of the newly developed blended wavelengths concept.

\section{Conflicts of Interest}

The authors declare no conflicts of interest regarding the publication of this paper.

\section{References}

[1] Ibrahimi, O.A., Avram, M.M., Hanke, C.W., Kilmer, S.L. and Anderson, R.R. (2011) Laser Hair Removal. Dermatologic Therapy, 24, 94-107. https://doi.org/10.1111/j.1529-8019.2010.01382.x

[2] Rao, J. and Goldman, M.P. (2005) Prospective, Comparative Evaluation of Three Laser Systems Used Individually and in Combination for Axillary Hair Removal. Dermatologic Surgery, 31, 1671-1676.

[3] Haedersdal, M. and Wulf, H.C. (2006) Evidence-Based Review of Hair Removal Using Lasers and Light Sources. JEADV, 20, 9-20. https://doi.org/10.1111/j.1468-3083.2005.01327.x

[4] Royo, J., Moreno-Moraga, J. and Trelles, M.A. (2017) Clinical Assessment of a New $755 \mathrm{~nm}$ Diode Laser for Hair Removal: Efficacy, Safety, and Practicality in $56 \mathrm{~Pa}-$ tients. Lasers in Surgery and Medicine, 49, 355-360.

[5] Tahiliani, S.T. and Tahiliani, H.S. (2016) Prospective Evaluation of the Safety and Efficacy of a 1060-nm Large Spot Size, Vacuum-Assisted Hair Removal Diode Laser System in Asian/Pacific Fitzpatrick's Skin Types IV-V Patients. Journal of drugs in dermatology, 15, 1427-1434.

[6] Passach, U., Wagner, J.A. and Passach, H. (2015) Novel 755-nm Diode Laser vs. Conventional 755-nm Scanned Alexandrite Laser: Side-by-Side Comparison Pilot Study for Thorax and Axillary Hair Removal. Journal Cosmetic and Laser Therapy, 17, 189-193. https://doi.org/10.3109/14764172.2015.1007062

[7] Courtney, E. and Goldberg, D.J. (2016) Clinical Evaluation of Hair Removal Using an $810 \mathrm{~nm}$ Diode Laser with a Novel Scanning Device. Journal of drugs in dermatology, 15, 1330-1333.

[8] Goldberg, D.J. (2018) One-Year Follow-Up Results of Hair Removal Using an 810 $\mathrm{nm}$ Diode Laser. Journal of Cosmetic Dermatology, 17, 775-778.

https://doi.org/10.1111/jocd.12757

[9] Scilletta, A. and Pompili, G. (2016) Laser Diode 800nm and Hirsutism in Darker Skinned Patients: Our Experience on 552 Women. Journal of Advanced Plastic Surgery Research, 2, 1-4.

[10] Koo, B., Ball, K., Tremaine, A.M. and Zachary, C.B. (2014) A Comparison of Two 
810 Diode Lasers for Hair Removal: Low Fluence, Multiple Pass Versus a High Fluence, Single Pass Technique. Lasers in Surgery and Medicine. https://doi.org/10.1002/lsm.22226

[11] Jo, S.J., Kim, J.Y., Ban, J., Lee, Y., Kwon, O. and Koh, W. (2015) Efficacy and Safety of Hair Removal with a Long-Pulsed Diode Laser Depending on the Spot Size: A Randomized, Evaluators-Blinded, Left-Right Study. Annals of Dermatology, 27, 517-522. https://doi.org/10.5021/ad.2015.27.5.517 